\title{
Tracing the origin and evolution history of methylation-related genes in plants
}

\author{
Liuling Pei, Lin Zhang, Jianying Li, Chao Shen, Ping Qiu, Lili Tu, Xianlong Zhang and Maojun Wang* (D)
}

\begin{abstract}
Background: DNA methylation is a crucial epigenetic modification, which is involved in many biological processes, including gene expression regulation, embryonic development, cell differentiation and genomic imprinting etc. And it also involves many key regulatory genes in eukaryotes. By tracing the evolutionary history of methylation-related genes, we can understand the origin and expansion time of these genes, which helps to understand the evolutionary history of plants, and we can also understand the changes of DNA methylation patterns in different species. However, most studies on the evolution of methylation-related genes failed to be carried out for the whole DNA methylation pathway.

Results: In this study, we conducted a comprehensive identification of 33 methylation-related genes in 77 species, and investigated gene origin and evolution throughout the plant kingdom. We found that the origin of genes responsible for methylation maintenance and demethylation evolved early, while most de novo methylation-related genes appeared late. The methylation-related genes were expanded by whole genome duplication and tandem replication, but were also accompanied by a large number of gene absence events in different species. The gene length and intron length varied a lot in different species, but exon structure and functional domains were relatively conserved. The phylogenetic relationships of methylation-related genes were traced to reveal the evolution history of DNA methylation in different species. The expression patterns of methylation-related genes have changed during the evolution of species, and the expression patterns of these genes in different species can be clustered into four categories.
\end{abstract}

Conclusions: The study describes a global characterization of DNA methylation-related genes in the plant kingdom. The similarities and differences in origin time, gene structure and phylogenetic relationship of these genes lead us to understand the evolutionary conservation and dynamics of DNA methylation in plants.

Keywords: DNA methylation, Gene origin, Duplication, Gene structure, Phylogenetic analysis

\section{Background}

The classical DNA methylation process is that the methyl group of S-adenosylmethionine is transferred to cytosines by different DNA methyltransferases, forming C5-methylcytosine (5-mC) [1]. In plants, DNA methylation is found at cytosines $(\mathrm{C})$ in three sequence contexts, including $\mathrm{CG}$, $\mathrm{CHG}$, and $\mathrm{CHH}(\mathrm{H}=\mathrm{A}$, T, or $\mathrm{C})$, which represents a difference from the predominant DNA methylation at the CG sequence context in animals [24]. DNA methylation is important for plant growth and development, and plays a role in genome imprinting,

\footnotetext{
* Correspondence: mjwang@mail.hzau.edu.cn

National Key Laboratory of Crop Genetic Improvement, Huazhong

Agricultural University, Wuhan 430070, Hubei, China
}

vernalization, response to biotic and abiotic stresses, and heterosis [1, 5-11].

In Arabidopsis thaliana, DNA methylation in all sequence contexts (CG, CHG, and $\mathrm{CHH}$ ) is established de novo by RNA-directed DNA methylation (RdDM) [5, 12-16]. The maintenance of methylation at CG and CHG sites depends primarily on DNA METHYLTRANSFERASE 1 (MET1) [5] and CHROMOMETHYLASE3 (CMT3) [17]. The methylation at $\mathrm{CHH}$ sites can't be maintained and must be re-established by both CHROMOMETHYLASE2 (CMT2) and RdDM [18]. In heterochromatin, de novo DNA methylation and maintenance require the action of DECREASE IN DNA METHYLATION1 (DDM1) [18]. DDM1 is one kind of nucleosome remodelers and can change the composition

(c) The Author(s). 2019 Open Access This article is distributed under the terms of the Creative Commons Attribution 4.0 International License (http://creativecommons.org/licenses/by/4.0/), which permits unrestricted use, distribution, and 
and position of the nucleosome, which makes chromatin more accessible to DNA methyltransferases [19]. Some active markers on histones are removed by JUMONJI 14 (JMJ14) [20], HISTONE DEACETYLASE 6 (HDA6) [21], UBIQUITIN-SPECIFIC PROTEASE 26 (UBP26) [22] and the repressed marker H3K9me2 is maintained by SUVH4 [23], which can maintain DNA methylation or the silencing state caused by DNA methylation.

The RdDM pathway relies on a specialized transcription machinery that is centered on two plant-specific homologs of RNA polymerase II (Pol II): Pol IV and Pol V [24-27]. In brief, the canonical RdDM process involves the following steps: Firstly, in the process of Pol IVdependent small interfering RNAs (siRNAs) biogenesis, the single-stranded RNA is transcribed by Pol IV followed by transforming into double-stranded RNA under the action of RNA-DEPENDENT RNA POLYMERASE (RDR2) [28] with the assistance of chromatin remodeler CLASSY 1 (CLSY1) [29]; Then, doublestranded RNA is cut into 24-nt siRNAs under the action of DICER-LIKE 3 (DCL3) [30]; The 3' end of the 24 nt siRNA is methylated by HUA ENHANCER 1 (HEN1) [31]; Finally, one strand of methylated 24-nt siRNA is loaded onto ARGONAUTE 4 (AGO4) [32]. Secondly, in the process of Pol V-mediated de novo methylation, the chromatin remodeler DEFECTIVE IN RNA-DIRECTED DNA METHYLATION 1 (DRD1) will open the DNA duplex and form a DDR complex with DEFECTIVE IN MERISTEM SILENCING 3 (DMS3) and RNADIRECTED DNA METHYLATION 1 (RDM1) to stabilize a loose chromatin state [33, 34], and MICRORCHIDIA 6 (MORC6) will assist in stabilizing the loose state [35, 36]; Pol V is recruited to the target site with the assistance of SU(VAR)3-9 HOMOLOG 2/9 (SUVH2/9) [37]; The AGO4 protein loaded with siRNAs is also recruited to the target site under the action of CARBOXY-TERMINAL DOMAIN (CTD) of Pol V and KOW DOMAIN-CONTAINING TRANSCRIPTION FACTOR 1 (KTF1), then siRNAs pair with the scaffold RNA transcribed by Pol V [38]; RDM1 can connect AGO4 protein with DOMAINS REARRANGED METHYLTRANSFERASE 1/2 (DRM1/2) to activate de novo establishment of DNA methylation. Thirdly, in the process of chromatin alteration, the INVOLVED IN DE NOVO 2 (IDN2) - IDN2 PARALOGUE (IDP) complex interacts with the SWI/SNF complex to change nucleosome positioning, thus making DNA more easily to be methylated [39].

Although DNA methylation is a relatively stable marker which can be inherited by the daughter cells after cell division, it's still reversible and dynamically regulated. In eukaryotes, the level of DNA methylation is not only related to the establishment and maintenance processes, but also related to the process of demethylation. The DNA demethylation is divided into passive demethylation and active demethylation. Passive demethylation is that the level of DNA methylation is reduced due to the loss of methylation maintenance in the process of DNA replication. Active demethylation is the process in which the 5-methylcytosine bases are replaced with non-methylated cytosines by DNA glycosylases/lyases and other enzymes [40]. In Arabidopsis, the main DNA glycosylases include Repressor of Silencing 1 (ROS1), DEMETER (DME), DME-like 2 (DML2) and DME-like 3 (DML3) [5]. These four demethylases can excise 5 -mC from all sequence contexts. Previous studies showed that ROS1 could conduct active DNA demethylation in all tissues [41], and $D M E$ was preferentially expressed in central cell and synergid of the female gametophyte [40, 42]. In addition, an EFFECTOR OF TRANSCRIPTION (ET) factor was characterized with a potential role in demethylation [43].

In recent years, many studies explored the evolution of methylation-related genes in plants. In hexaploid wheat, all MET1 genes were divided into three categories, and these genes were derived from whole genome duplication within the grass family and gene duplication which occurred specifically in the Triticeae tribe [44]. The discovery of DNA methyltransferases in different plants and fungi indicated that MET1 was highly conserved [11]. The evolution analysis of RNA polymerase subunits showed that genes encoding Pol IV and Pol V evolved rapidly relative to Pol II. An analysis of genes in the RdDM pathway in 15 species showed that RdDM appeared in early land plants [45]. Recently, the origin of the CMT family was deduced in terrestrial plants by using data in 443 species [46].

Here, we explored phylogenetic relationships of methylation-related genes on a rather long evolutionary scale from algae to flowering plants. We identified 33 methylation-related genes in 77 species, and explored the origin and evolution of genes in de novo methylation, maintenance methylation and demethylation processes. The gene structural changes were investigated by an exon-intron structure analysis, and the functional changes were studied by a domain analysis. In addition, a phylogenetic analysis showed that these methylationrelated genes were divided into three categories, i.e., early plants, monocotyledon plants and dicotyledon plants.

\section{Results}

The inconsistency in the origin of methylation-related genes

The methylation-related genes have been comprehensively characterized in A. thaliana. In this study, all these genes were divided into five categories including eight groups. The five categories included genes 
primarily functioning in de novo DNA methylation, methylation maintenance, nucleosome remodeling, histone modification and demethylation (Fig. 1). The canonical RdDM process of de novo DNA methylation includes three steps: Pol IV-dependent siRNA biogenesis, Pol V-mediated de novo methylation and chromatin alteration. We identified methylation-related genes for all these categories in 77 species involving of algae and plants (Additional file 4: Table S1). These algae include five species, and plants include species from bryophyte, fern, gymnosperm, basal angiosperm, monocot and dicot. We summarized the content of methylationrelated genes in each species (Additional file 5: Table S2).

In each of the five categories, we calculated the ratio of the number of species containing methylation-related genes to the total number of species, and explored the evolution of these genes from algae to higher flowering plants (Fig. 1). In the RdDM process, we investigated 5 genes required for Pol IV-dependent siRNA biogenesis. Of them, only HEN1 is found in algae, and some genes in the bryophytes, ferns, monocots and dicots are missing. There are 9 genes involved in Pol V-mediated de novo methylation. SUVH2 is absent in basal angiosperm, and is also absent in $71 \%$ of dicots, whereas it is found in all monocots. There are 2 genes involved in the chromatin alteration process, they are absent in the algae. For RNA polymerases, we explored the NUCLEAR RNA POLYMERASE D1 (NRPD1), NRPE1, NRPD2/E2,
NRPD4/E4, and NRPE5. Interestingly, all the five subunits are not found in algae. The methylation of the $\mathrm{CHH}$ site is found to be re-established by the process of de novo DNA methylation during each cell cycle, while de novo DNA methylation of the $\mathrm{CHH}$ site includes $\mathrm{RdDM}$ and another RdDM-independent way [12]. The latter requires the participation of CMT2 which is absent in algae. Collectively, we conclude that only a few genes involved in de novo DNA methylation appeared in algae, however, these genes became widespread from bryophytes to higher plants. Therefore, the de novo DNA methylation process may appear after divergence of chlorophyta and embryophyta.

In the process of maintenance of DNA methylation, different enzymes work in different ways. The methylation of CG requires the participation of MET1 which is basically present in all these species, including four of the five lower algae species. The methylation of $\mathrm{CHG}$ requires the participation of $C M T 3$ which is also basically present in these studied species. Therefore, the maintenance of CG and CHG methylation may derive from the common ancestors of chlorophyta and embryophyta. The nucleosome remodeler $D D M 1$ which is essential for mediating methylation in the heterochromatin region, is basically present in all the studied species. The time of its occurrence is consistent with that of DNA methyltransferases (MET1 and CMT3). There are 4 genes (JMJ14, UBP26, HDA6 and SUVH4) involved in the histone modification process, they all are found in the

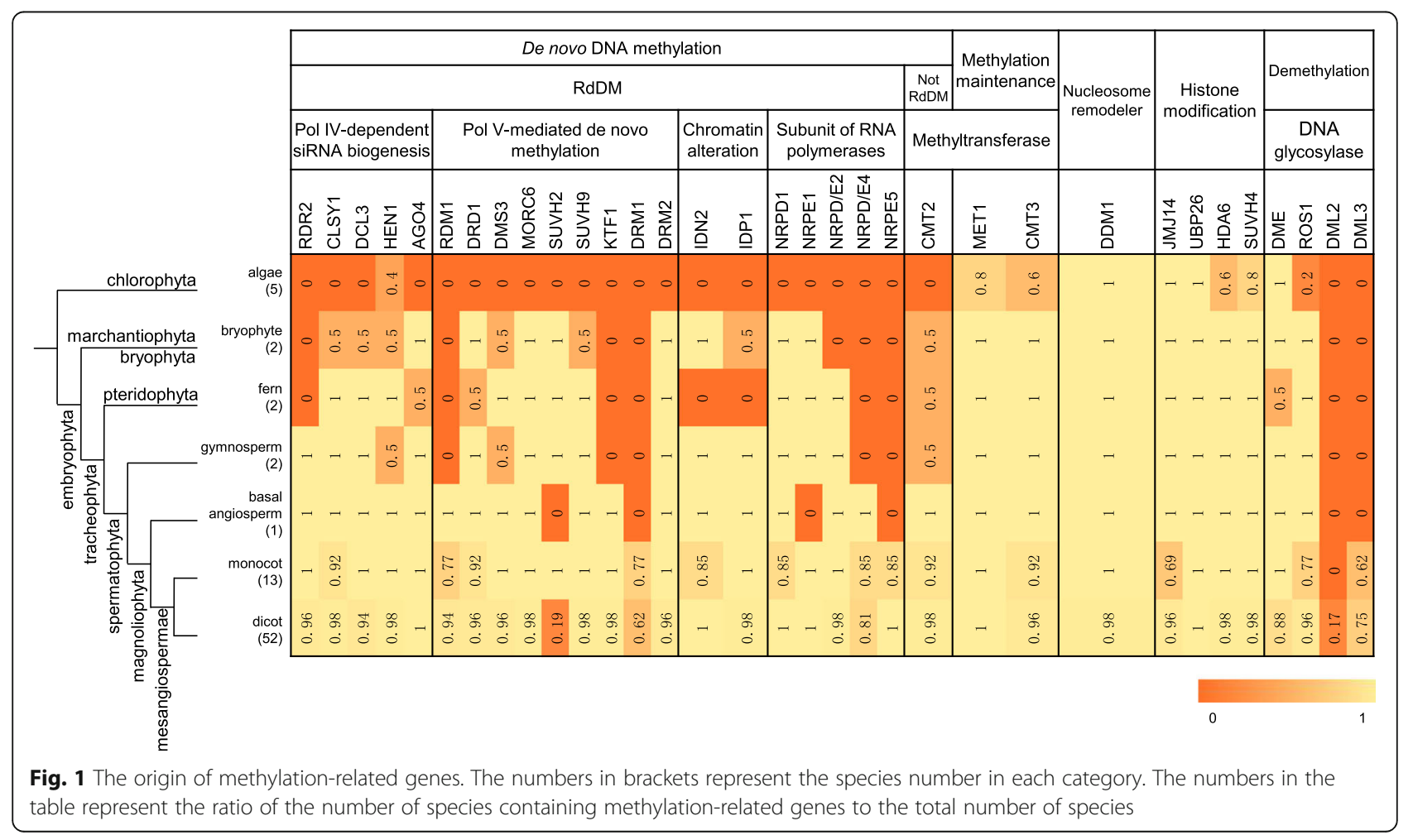


algae. These genes are important components involved in histone modications, thereby they influence DNA methylation and can affect phenotypic traits such as flowering time and drought tolerance of plants [47-50].

For the DNA demethylation process, active demethylation is mediated by four types of DNA glycosylases (DME, ROS1, DML2 and DML3). The previous studies showed that $D M E$ was found only in dicots [42], but our results indicated that $D M E$ and $R O S 1$ were present from algae to higher flowering plants. $D M L 2$ can be seen only in dicots with a relatively small proportion (17\%). An analysis of the distribution of DML2 in different species showed that it was only present in cruciferous plants (Additional file 5: Table S2). DML3 was present in dicots and monocots. These results showed that DNA demethylation might have originated from the common ancestor of chlorophyta and embryophyta, while $D M L 2$ is limited in cruciferous plants and $D M L 3$ is limited in dicots and monocots.

\section{The abundance and duplication of methylation-related genes}

To explore how methylation-related genes evolved, we investigated the abundance and duplication patterns of methylation-related genes in different species. Firstly, we summarized the abundance of methylation-related genes in different species (Additional file 5: Table S2). A large number of gene expansion events were observed. In 28 diploid species, such as Setaria italica, Brachypodium distachyon, Fraxinus excelsior, Malus domestica, Lotus japonicus, Glycine max, Populus trichocarpa and Citrus sinensis, the number of genes containing multiple copies was significantly larger than that in the other 43 diploid species (ANOVA, $p<0.01$ ) (Additional file 5: Table S2). Among the six polyploid species, including five tetraploids (Panicum virgatum, Nicotiana tabacum, Gossypium hirsutum, Gossypium barbadense, Brassica napus) and one hexaploid (Triticum aestivum), the number of methylation-related genes was normalized by dividing them at the diploid level. The result showed that the number of genes containing multiple copies was relatively larger in $P$. virgatum, $T$. aestivum and $B$. napus (ANOVA, p < 0.01) (Additional file 6: Table S3).

Gene absence is also observed for some methylationrelated genes. In algae, there is a notable absence of methylation-related genes (Additional file 5: Table S2). There are 23 methylation-related genes that are not present in all five algae species. It is only found that 4 methylated-related genes, JMJ14, UBP26, DDM1 and $D M E$, were present in all 5 algae. The absence rate of methylation-related genes is much larger in advanced plants. In monocots, 1 to 10 genes were missing. In dicots, there are 48 species that had gene absence events. In the three Arabidopsis species, the methylation-related genes in A. thaliana and A. lyrata were conserved, while in the A. halleri, 4 genes (RDM1, SUVH9, HDA6 and $N R P D / E 2)$ were absent. In Solanum tuberosum, the absence of genes was greatly pronounced, with 10 of the 33 genes were missing.

The expansion of methylation-related genes may be related to whole genome duplication (WGD) and tandem duplication. For example, the genome duplication event of G. max that occurred approximately 13-59 million years ago, contributed to the formation of multiple copies for nearly $75 \%$ of genes [51]. About 50 million years ago, there was a WGD event in $M$. domestica, which increased gene number significantly [52]. Two IDN2 genes in Gossypium raimondii, Gorai.010G201400 and Gorai.010G201300, were evolved from tandem duplication. Here, we analyzed the evolutionary patterns of methylation-related genes in Oryza sativa, Solanum lycopersicum, Vitis vinifera, Amborella trichopoda, Cucumis sativus and Phaseolus vulgaris (Additional file 7: Table S4). Through the analysis of WGD and tandem duplication information of these five species [53, 54], we found that both duplication patterns contributed to gene expansion in these species. Two copies of the MET1 gene in O. sativa were evolved from WGD. In three copies of AGO4 gene in P. vulgaris, Phvul.008G206600 and Phvul.006G021200 were evolved from WGD, Phvul.008G206600 and Phvul.008G206500 were evolved from tandem duplication. The similar phenomenon can also be found in S. lycopersicum, V. vinifera, A. trichopoda and C. sativus.

We used 17 of 77 species for a gene collinearity analysis, including four early plants, four monocots and nine dicots. In each methylation process, we carried out a collinear analysis of some genes (Fig. 2 Additional file 1: Figure S1). A total of 10 methylation-related genes were analyzed. The collinearity of chromosomal regions which contained methylation-related genes indicated that these genes were the products of WGD or segment duplication. For AGO4, DMS3 and other genes, some chromosomal segments had better collinearity in the same species. We also compared the collinearity between different species. There is no collinearity between the early plants and the monocots or dicots for most genes, and there is also no collinearity between monocots and dicots for most genes. In different early plant species, chromosomal collinearity is not observed. In monocots, the collinearity of Zea mays, Sorghum bicolor, and Oryza sativa is found for most genes. In dicots, the collinearity of most genes is observed, however, some genes in A. thaliana, Citrullus lanatus, S. lycopersicum and Carica papaya are in poor collinearity. These data suggest that genome evolution has an effect on disrupting chromosomal collinearity in different species in terms of methylation-related genes. 

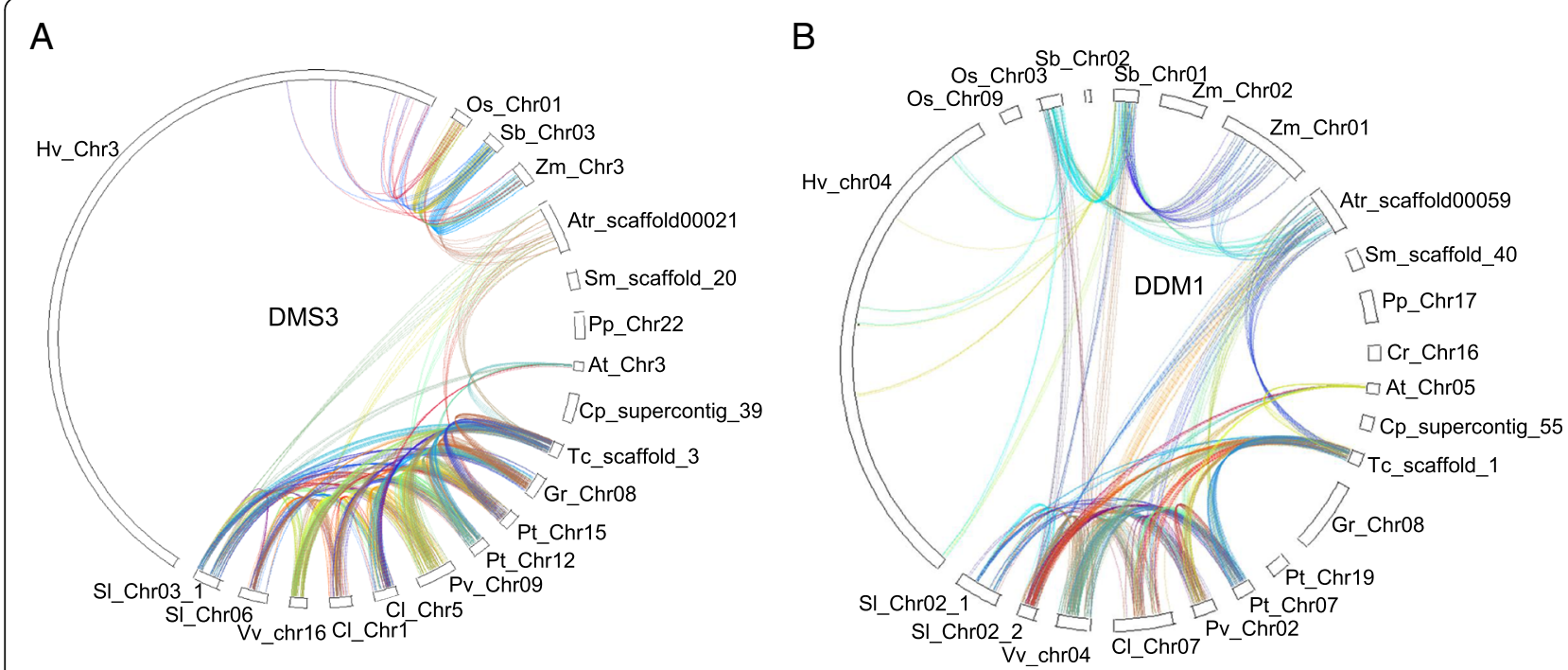

Fig. 2 Collinear analysis of chromosome fragments containing 20 adjacent genes upstream and downstream of methylation-related genes. a The collinearity of DMS3 genes in different species. $\mathbf{b}$ The collinearity of DDM1 genes in different species. The species names are Chlamydomonas reinhardtii (Cr), Physcomitrella patens (Pp), Selaginella moellendorffii (Sm), Amborella trichopoda (Atr), Zea mays (Zm), Sorghum bicolor (Sb), Oryza sativa (Os), Hordeum vulgare (Hv), Solanum lycopersicum (SI), Vitis vinifera (Vv), Citrullus lanatus (Cl), Phaseolus vulgaris (Pv), Populus trichocarpa (Pt), Gossypium raimondii (Gr), Theobroma cacao (Tc), Carica papaya (Cp), Arabidopsis thaliana (At)

\section{The conservation and change of gene structures of methylation-related genes}

To explore how gene structure has changed during evolution, we used 18 representative species for an exonintron structure analysis. We started this analysis by examining exon-intron structure of these methylationrelated genes at the species level (Additional file 8: Table S5). Generally, gene length and intron length varied largely (Additional file 8: Table S5A-B, S5G-H), but the exon number and exon length of most genes were conserved (Additional file 8: Table S5C-F). The correlation analysis indicated that the change of gene length was primarily due to the change of intron length $(\mathrm{r}=0.79$, $p<0.01$ ). For example, in $A$. trichopoda, $Z$. mays, $A$. thaliana and $V$. vinifera, gene length and intron length of most methylation-related genes changed a lot, but the exon length varied little. Interestingly, in C. reinhardtii, $P$. abies and $C$. papaya, the gene length and intron length of most methylation-related genes varied a lot, but the exon length and exon number of some methylation-related genes also had some changes.

Then, the exon-intron structure was studied at the functional domain level of protein sequences (Fig. 3 and Additional file 2: Figure S2). First, five genes were studied which were involved in de novo DNA methylation (Additional file 2: Figure S2A-E), including $R D R 2$, CLSY1, DCL3, HEN1 and AGO4 in the process of Pol IV-dependent siRNA biogenesis. The exon-intron structure of AGO4 was conserved, and the changes of exonintron structure of the other four genes were large, mostly because of intron changes. The domain structure of these genes was basically conserved. In the Pol Vmediated de novo methylation (Fig. 3a, Additional file 2: Figure S2F-M), the changes in exon-intron structure of these genes were notable except for KTF1. The domain structure of RDM1, DRD1, MORC6 and KTF1 was highly conserved from early plants. It is found that DMS3 only contains low complexity regions and coiled coil regions. SUVH $2 / 9$ had SRA, PreSET and SET domains in spermatophyte, but both genes had only one of these three domains in early plants. In the chromatin alteration process (Additional file 2: Figure S2 N-O), the gene and intron length of IDN2 and IDP1 changed a lot, but their protein domains were highly conserved. In the subunits of RNA polymerases (Additional file 2: Figure S2 P-T), the changes of exon-intron structure for NRPD1, NRPD/E4 and NRPE5 were mainly because of intron changes. Their functional domains were conserved with key domains existing in the most ancestral species. We also found that in early plants ( $P$. abies and A. trichopoda), the domain structure of some key genes changed greatly. A similar trend was observed in monocots $O$. sativa and $H$. vulgare and dicot $C$. papaya. In the process of de novo $\mathrm{CHH}$ methylation (Additional file 2: Figure S2U), the exon-intron structure of CMT2 changed a lot. The CMT2 in C. papaya was short and contained only a partial domain, and CMT2 in P. abies and $H$. vulgare were short and did not contain domains that existed in other species.

Second, we investigated genes responsible for maintenance of DNA methylation (Fig. 3b, Additional file 2: Figure S2V). The exon-intron structure and domains of 


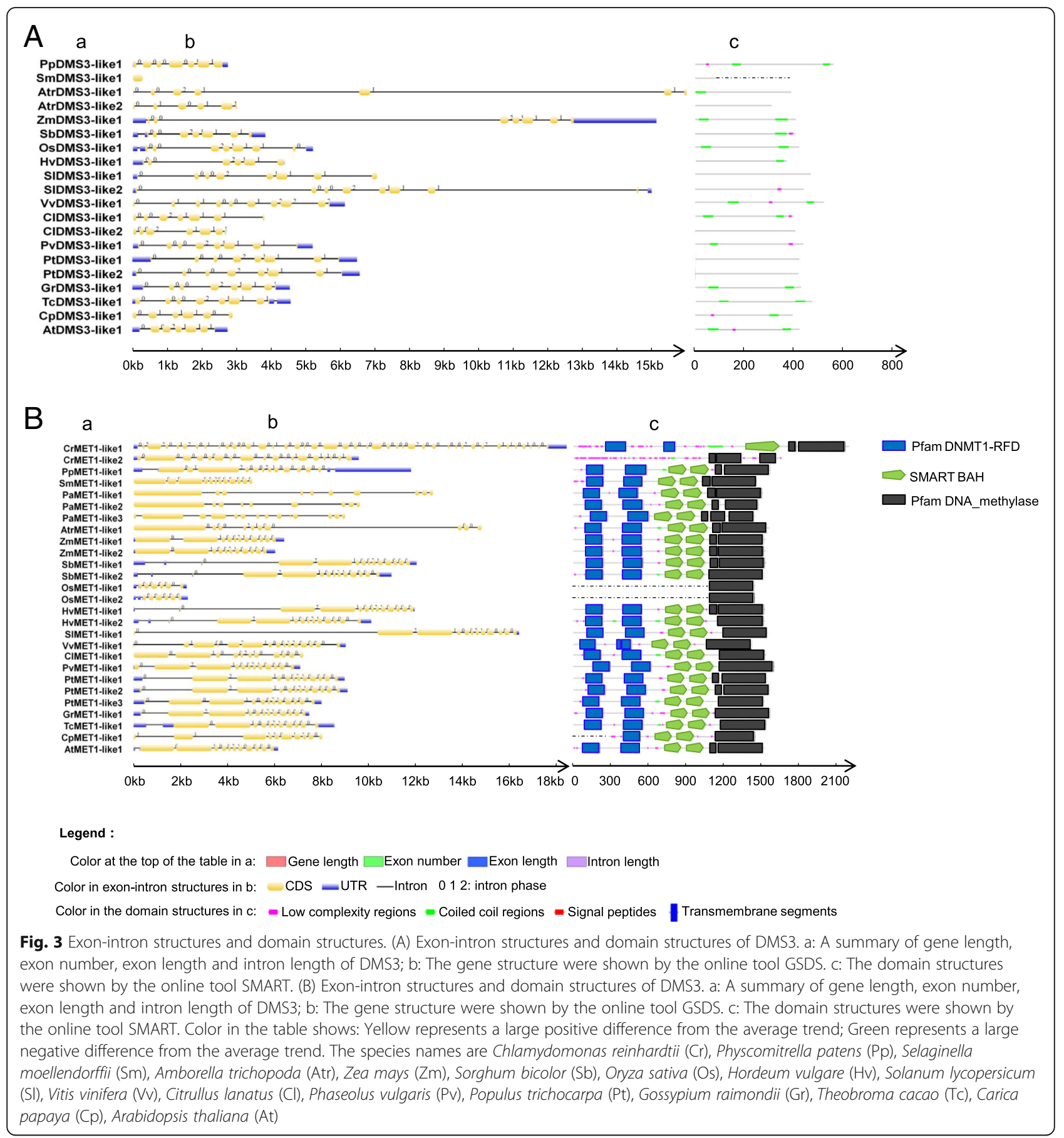

two genes (MET1 and CMT3) were generally conserved. However, for two duplicated MET1 genes in rice, only the DNA_methylase domain was found in each copy, and the protein sequences were short. In C. reinhardtii, CMT3 contained some peptide segments with lowcomplexity, but lacked the BAH and CHROMO domains. In P. abies, these domains of CMT3 were absent. The domains of CMT3 in rice were also different from that in Arabidopsis. As for nucleosome remodeling factor DDM1 (Additional file 2: Figure S2W), the exon-intron and domain structures were highly conserved in higher plants. In the histone modification process (Additional file 2: Figure S2X-Z, S2AA), the exon-intron structure of genes changed a lot, but their protein domains were highly conserved from early plants.

Finally, we investigated genes responsible for DNA demethylation (Additional file 2: Figure S2BB-DD). The 
exon-intron and domain structures of DME, ROS1 and $D M L 3$ were conserved. There were only a few exceptions. The exon length of $D M E$ in $C$. reinhardtii was long. For domain structures, $D M E$ in $C$. reinhardtii contained a large number of low-complexity regions in addition to the partial domain of $A$. thaliana. The protein sequences of DME and ROS1 in P. abies were short and contained only a partial domain. ROS1 in C. papaya was short and did not contain any domains.

In summary, the exon-intron structure and functional domains varied greatly in the early plants, but they were conserved for most genes in monocots and dicots. Of note is the observation that a few domains of some genes in monocots $H$. vulgare and $O$. sativa varied greatly, and the dicot species $C$. papaya had considerable changes in the domains of most genes. Genes with domain changes had notable changes in exons, which is consistent with the overall changes of the exon- intron structure at the species level (Additional file 8: Table S5).

\section{The phylogenetic relationship and purifying selection of methylation-related genes}

The identification of genes in more than 77 species has led to investigate the origin of methylation pathways. Through exon-intron and domain structure analysis, we have known that although the length of genes varied greatly, but the functional structures were relatively conserved. Next, we sought to explore the phylogenetic relationship of methylation-related genes (Fig. 4 and Additional file 3: Figure S3).

At first, we studied de novo DNA methylation-related genes (Fig. 4a-b, Additional file 3: Figure S3A-O). The de novo DNA methylation process involves many genes, and a phylogenetic analysis categorized them into three groups, including early plants, dicots and monocots. A
A

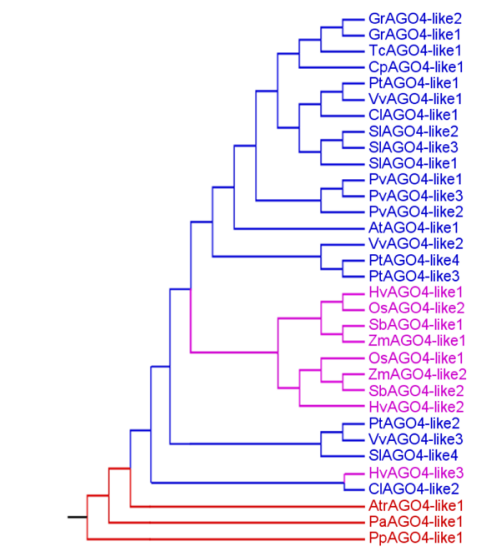

C

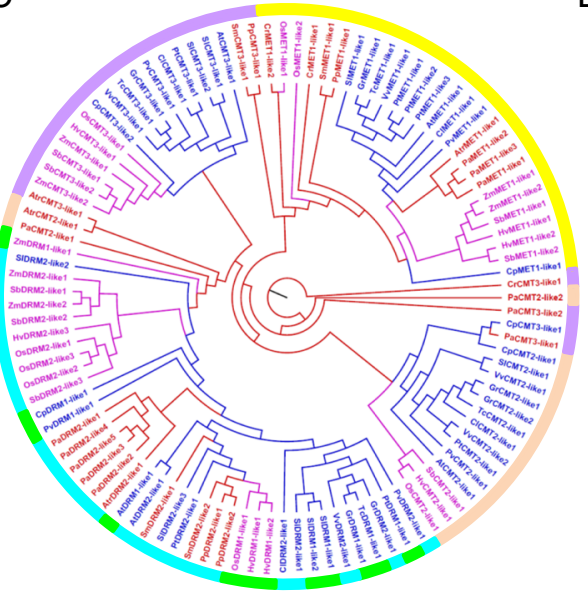

Color in circle of C: MET1 $\quad$ CMT2 $\quad$ CMT3 DRM1 DRM2

Color in all tree: $\neg$ Early plants $-\ulcorner$ Monocots $\leftarrow$ Dicots

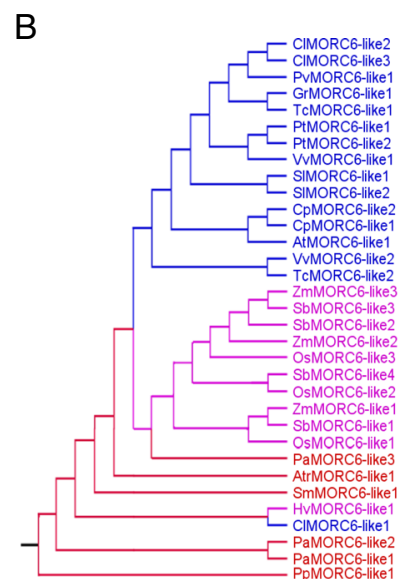

D

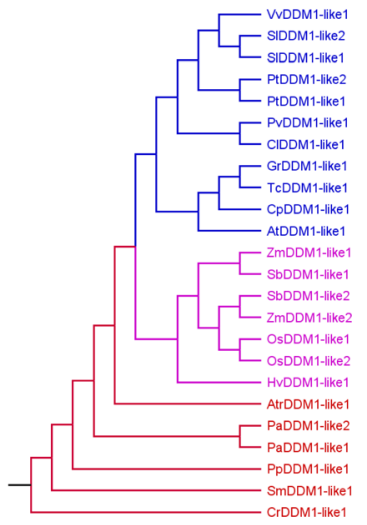

Fig. 4 The evolutionary tree of methylation-related genes constructed by neighbor joining method in 18 species. a The evolutionary tree of AGO4 (ARGONAUTE 4) gene. b The evolutionary tree of MORC6 (MICRORCHIDIA 6) gene. c The evolutionary tree of methyltransferases. d The evolutionary tree of DDM1 (DECREASE IN DNA METHYLATION1) gene 
detailed analysis of these data showed that most methylation-related genes with multiple homologs tended to cluster in different branches in monocots, however most of these genes tended to gather in a single branch in dicots. We hypothesize that methylationrelated genes in monocots may have been duplicated prior to the divergence of monocots and dicots, and possibly most of the genes in dicots tend to duplicate after this divergence event. The MORC6 genes in monocots are clustered into three branches, while the MORC6 genes in dicots C. lanatus, P. trichocarpa, C. papaya and $S$. lycopersicum were clustered in one branch. A similar observation was obtained for AGO4,DCL3, DRD1 and IDP1. Because both NRPD1 and NRPE1 came from $N R P B 1$ and the sequence similarity between NRPD1 and NRPE1 was very high, we combined NRPD1 and NRPE1 subunits together to explore their evolutionary relationship. In early plants, NRPD1 and NRPE1 were clustered together, but they were clustered into two branches in angiosperms, which suggested that NRPD1 and NRPE1 might be divergent from the ancestors of monocots and dicots.

We then studied those genes responsible for maintenance of DNA methylation (Fig. 4c). In order to explore a full phylogenetic relationship of these methyltransferases, we constructed an evolutionary tree by combining them together with those responsible for de novo methylation. It can be seen that MET1, CMT2 and $C M T 3$ were well clustered together, and the DRM1 and $D R M 2$ were clustered into two branches in monocots but clustered together in dicots and early plants. As for the nucleosome remodeling factor DDM1, we found that all the DDM1 genes in different species were clustered into three branches (Fig. 4d). In the histone modification process (Additional file 3: Figure S3P-S), with some exceptions, JMJ14, UBP26, HDA6 and SUVH4 are basically clustered into three branches.

Finally, we analyzed demethylation-related genes. In the monocots and dicots, most DNA glycosylases were clustered separately into the DME branch, the ROS1 branch or the DML3 branch, but they were clustered together in the early plants (Additional file 3: Figure S3T). This result suggests that the ancestral demethylationrelated genes might be differentiated into different demethylation-related genes after the divergence of monocots and dicots.

In addition, we investigated the selection pressure of methylation-related genes by calculating the ratio of nonsynonymous substitutions per nonsynonymous site (Ka) to synonymous substitutions per synonymous site (Ks) (omega) for each homologous gene pair in nine dicots, with reference to genes in A. thaliana. We found that all genes were under negative selection (Fig. 5). This indicated that these genes were highly conserved during the evolution of dicotyledonous plants, which also reflected the importance of DNA methylation for dicotyledonous plants.

\section{Divergent expression patterns of methylation-related genes in different species}

On the basis of tracing evolution history of methylationrelated genes from early plants to higher plants, we next aim to explore how the methylation-related genes have evolved at the expression level in different species. We selected 10 species for analysis of expression patterns, including algae, bryophyte, fern, basal angiosperm, dicot and monocot. In three early plants - $C$. reinhardtii, $P$. patens and $S$. moellendorffii, samples from the whole plant were used; in basal angiosperm A.trichopoda, 3 dicots and 3 monots, data from the leaf tissue were used. The normalized expression level of gene (TPM) was shown in the form of heatmap (Fig. 6).

First of all, we compared the expression patterns of methylation-related genes at the species level. We can see that the early plants, monocots and dicots were clustered into one branch, respectively. This indicated that the expression patterns of methylation-related genes might have changed during the evolution from early plants to higher plants, as well as during the differentiation of monocots and dicots. In the evolution of these genes, there were changes not only in duplication pattern and gene structure, but also in gene expression patterns, which provides an idea for us to study the origin and evolution of other genes. From the gene level, we can see that the expression patterns of these genes can be clustered into four categories. Here, we mainly analyzed the expression pattern of angiosperms. In cluster III and IV, the expression levels were high, which may reflect the conservation of gene expression in species evolution, and also explain the importance of these genes in plant growth and development. UBP26 and HDA6 are important components involved in histone modications, thereby influence DNA methylation and affect phenotypic traits [47-50]. DRM2 is the key gene in RdDM pathway [5, 12-16]. In cluster I and cluster II, the expression levels of these genes were low.

\section{Discussion}

DNA methylation is common in the entire eukaryotic species and is essential for plant growth and development, which can cause gene and transposon silencing and genomic imprinting, and regulate other biological processes $[1,5-10]$. The methylation process has been systematically studied in A. thaliana. In this study, we focus on the origin and evolution of methylation-related genes in the whole plant kingdom. 

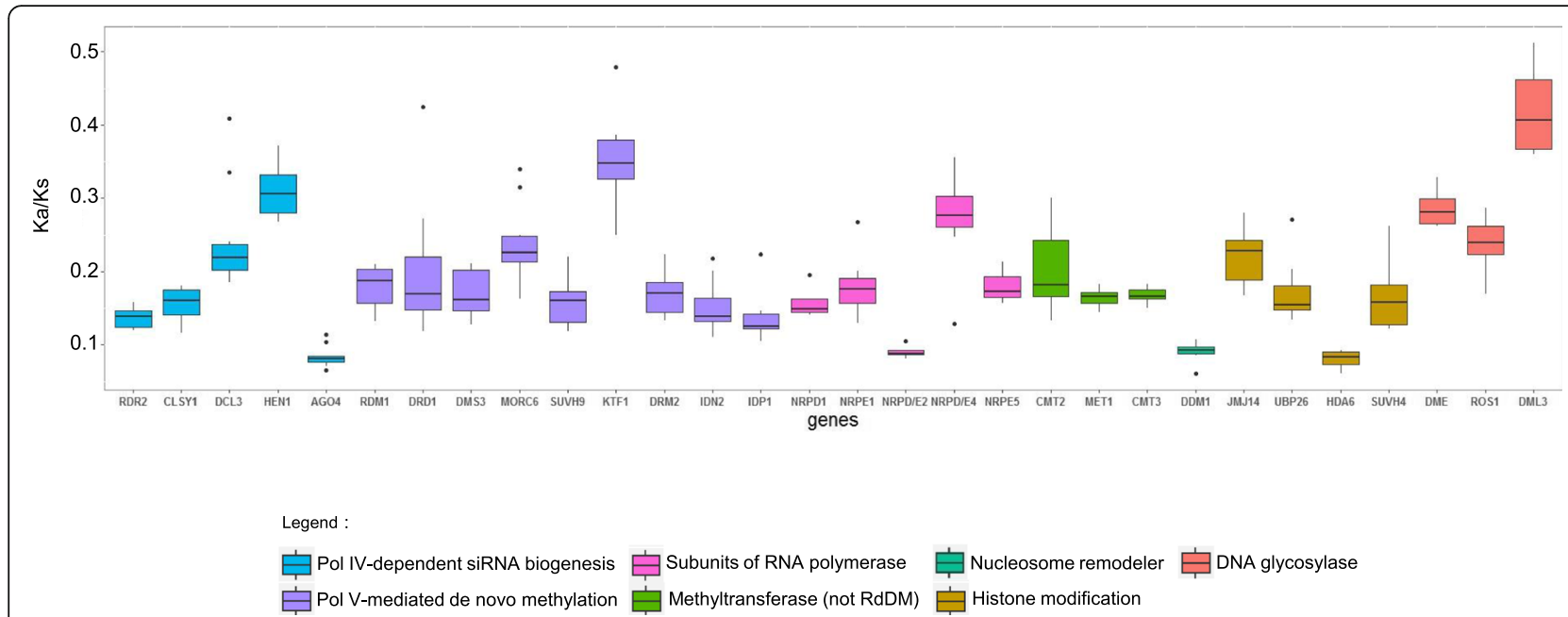

Fig. 5 The distribution of Ka/Ks values of methylation-related genes. The Ka and Ks values were computed using the PAML program. The Ka/Ks value less than 1 indicates that the gene is under negative selection

\section{The origin of methylation-related genes}

The de novo DNA methylation process may be established from the common ancestor of bryophyte and tracheophyta after divergence of chlorophyta and embryophyta (Fig. 1). The maintenance of CG and CHG methylation can be derived from the common ancestor of chlorophyta and embrypphyta. Hypothetically, DNA methylation may be established firstly, and then maintenance of methylation starts to work on the basis of the established methylation, i.e., de novo methylation may appear earlier than maintenance. But, in our study, genes associated with the maintenance of CG and CHG methylation appeared earlier than most genes for de novo methylation. DNA demethylation may have originated from the common ancestor of chlorophyta and embryophyta, which is similar to the maintenance of CG and CHG methylation. But DML2 was identified in cruciferous plants, which may come from the evolution of the $D M E$ after crucifer differentiation, and $D M L 3$ was limited in mesangiospermae, which may come from $D M E$ after divergence of mesangiospermae and basal angiosperm. In other words, the mesangiospermae and the cruciferous plants may have also evolved new demethylation processes which depends on DML3 and

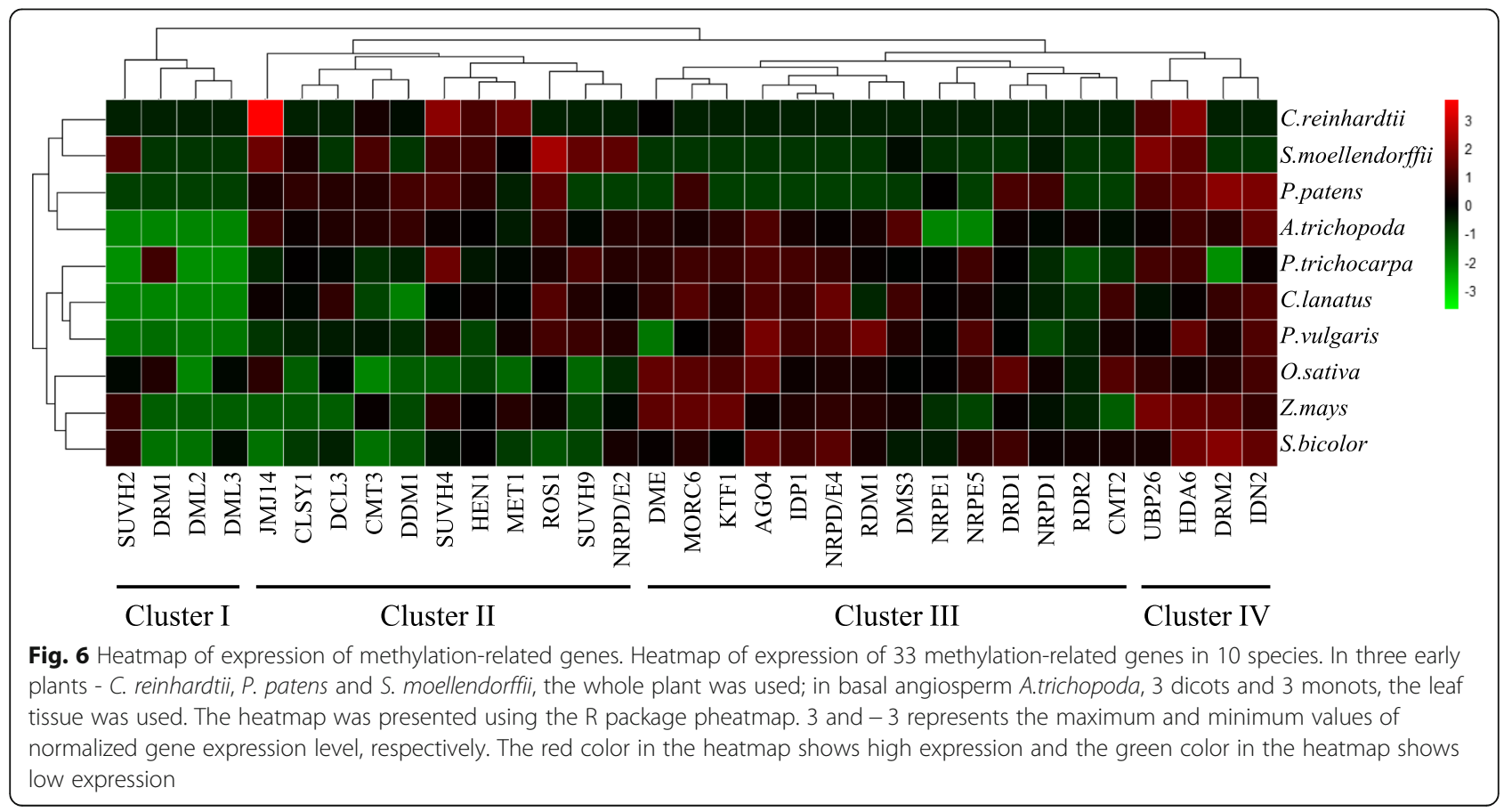


$D M L 2$. It requires further investigation to know whether the presence of these two enzymes means that the demethylation process appears in cruciferae superior to other species. The symmetric methylation (CG and CHG) can be maintained by MET1 and CMT2 which appeared earlier, and most de novo methylation-related genes appeared late. And there is no systematic comparison of the conservation of symmetric methylation with other methylation-related genes. In the following studies, we can explore whether symmetric methylation can be maintained easier and is therefore evolutionarily conserved.

Histone modification interacts with DNA methylation in plants. H3K9me2 is a silent mark, and $70 \%$ of $\mathrm{RdDM}$ targets are modified by H3K9me2 [55]. SUVH4 maintains H3K9me2 and thereby contributes to DNA methylation. Acetylation and ubiquization of $\mathrm{H} 2 \mathrm{~B}$ and H3K4me are active markers. The active marks of RdDM targets need to be removed in order to maintain DNA methylation and the silencing state caused by DNA methylation [20-23]. These four histone modifications evolved earlier than genes for de novo methylation, indicating that histone modification might affect the growth and development of plants in some other ways before de novo methylation.

\section{The absence of methylation-related genes}

The gene absence was common in plants (Additional file 5: Table S2). Of the 77 species, 73 species had gene absence. The CMT3 genes were reported to have been lost in two angiosperms (Eutrema salsugineum and Conringia planisiliqua) [56]. In algae, the widespread gene absence may be due to their later evolution; in higher plants, such as monocots and dicots, these common absence events pointed to the possibility that epigenetic genes might be lost or gained in individual lineages of angiosperms. The effects of the absence of these genes on the apparent modification of plants need to be further explored.

\section{The exon-intron structure and the methylation level of species}

In this study, we have found that most species exhibited conserved exon length and number for methylationrelated genes. Simultaneously, the domain sequences which were closely related to the function of genes were also highly conserved. In C. papaya, the exon-intron structure and domain changed greatly. One reason for this may be the relatively poor quality of the genome assembly, and the other is that these genes indeed have evolved dramatically during evolution. The exon-intron structure and domains of a number of genes changed greatly in $O$. sativa, such as MET1 (Fig. 3). MET1 in rice had only the DNA methylase domain and had no BAH and DNMT1-RFD domains, but MET1 in the other species contained all three types of domains. Based on the whole genome methylation data in $O$. sativa and other species, we could see that CG methylation content in $O$. sativa was higher than $A$. thaliana, T. cacao and $V$. vinifera [57], which indicated that the change in MET1 did not result in the overall decrease of CG methylation level in O. sativa. The reason why MET1 lacked the key domain and the CG methylation content remained at a high level requires further exploration.

\section{Conclusions}

DNA methylation is one of the most common modifications in plants, which involves the dynamic balance of de novo methylation, maintenance methylation and demethylation. The origin time of different methylationrelated genes is not consistent, which reflects the evolutionary complexity of DNA methylation in plants. The conservation in domain structure of gene sequences and negative selection of these genes reflect the conservation of the methylation process, which points to the functional importance of DNA methylation to plants.

\section{Methods}

\section{Identification of methylation-related genes}

The methylation-related genes in A. thaliana were retrieved from the TAIR (www.arabidopsis.org/) database. The protein sequences and coding sequences (CDS) of the other species, as well as the General Feature Format 3 (GFF3) and annotation files were downloaded from the websites shown in Additional file 9: Table S6. The protein sequence of methylation-related genes in $A$. thaliana were used as queries to identify homologous genes in the other 76 species using blastp program (sequence coverage $>30 \%$, identity $>30 \%$ and E-value cutoff $1 \mathrm{e}-3$, other parameters are default). At the same time, each candidate homologous gene was manually checked in combination with annotation file of each species. The partial genes that might result from uncomplete genome assembly were excluded manually after sequence alignment. The species taxonomy tree was modified according to the information in the Taxonomy database of NCBI (https://www.ncbi.nlm.nih.gov/ taxonomy/).

\section{Collinearity analysis}

The chromosome location of methylation-related genes in each genome was obtained according to the GFF3 file. When homologous methylation-related genes were located in the same chromosome with no more than one intervening gene, these genes were defined as tandem duplication genes. We used the method as described in Maher et al. to identify large-scale duplication events [58]. If two genes were located in the same duplication 
block, the protein sequences on their flanks were highly similar at the amino acid level. Therefore, we located the methylation-related genes on the genome and used this location as the initial anchor site to obtain 20 proteincoding genes upstream and downstream of each site respectively [58]. This chromosomal regions containing 41 protein-coding genes were selected for collinearity analysis using MCScanX (blastp E-value cutoff 1e-10) [59].

\section{The exon-intron and domain structure analysis}

The exon-intron structure information of methylationrelated genes in every species was extracted from the GFF3 file using an in-house Perl script. Gene names used in gene structure analysis and phylogenetic analysis were shown in Additional file 10: Table S7. When multiple homologous genes in other species corresponded to one methylation-related gene in A. thaliana, the gene with the longest length was used to calculate the gene length, exon number, exon length and intron length. The diagrams of the exon-intron structure were drawn using the online tool GSDS (http://gsds.cbi.pku.edu.cn/). The protein domain structures were drawn using the online tool SMART (http://smart.embl-heidelberg.de/). The SMART and Pfam databases were used for domain prediction. In the exon-intron structure, the gene length values were averaged after removing two extreme values. When the gene length is more than 1.5-fold of the average value, the length is judged to be long. When the gene length is less than half of the average value, the length is judged to be short. These two values are called extreme values. When the value is between 0.5 and 1.5fold of the average value, the length is judged to be moderate. Exon number, exon length and intron length are treated similarly.

\section{Phylogenetic analysis}

The protein sequences of all methylation-related genes were aligned using Clustal X (version 2.0) [60]. Phylogenetic analysis was conducted using PHYLIP software with the neighbor-joining (NJ) method (bootstrap value 1000) [61]. The Figtree (version 1.4.3) software was used to display the phylogenetic tree.

\section{The estimation of selection pressure of methylation- related genes}

Clustal X (v2.0) was used to conduct multiple alignment of protein sequences of methylation-related genes, which was guided by PAL2NAL software with the nogap parameter [62]. The yn00 procedure in PAML package was used to calculate the ratio of nonsynonymous substitutions per nonsynonymous site (Ka) to synonymous substitutions per synonymous site (Ks) for each homologous gene pair [63]. According to the definition of $\mathrm{Ka} / \mathrm{Ks}$, values less than one represent negative or purifying selection, while values greater than one represent positive selection. The saturation effect was ruled out by removing gene pairs with $\mathrm{Ks}>2.5$.

\section{The expression analysis of methylation-related genes}

The RNA-Seq data in different species were collected from the NCBI Sequence Read Archive (SRA) database (Additional file 11: Table S8). In three early plants - $C$. reinhardtii, $P$. patens and $S$. moellendorffii, the whole plant was used; in basal angiosperm A.trichopoda, 3 dicots and 3 monots, the leaf tissue was used. Firstly, these raw reads were trimmed using trimmomatic- 0.32 software [64]. Then, the clean data were mapped against the genomes using HISAT2 software (version 2.1.0) [65]. Finally, the StringTie software (version 1.3.4) was used to calculate the expression level for each gene normalized as Transcripts Per Million (TPM) [66]. When a methylation-related gene was absent in a species, the gene can not be transcribed, we recorded the expression of the gene as 0 ; When there are multiple homologous copies in a species, the sum of the expression of homologous copies is recorded as the expression level in a species. The normalized expression level of gene (TPM) was shown in the form of heatmap using the $\mathrm{R}$ package pheatmap.

\section{Additional files}

Additional file 1: Figure S1. Collinear analysis of chromosome fragments containing 20 adjacent genes upstream and downstream of methylation-related genes. (A) The collinearity of RDR2 genes in different species. (B) The collinearity of AGO4 genes in different species. (C) The collinearity of MORC6 genes in different species. (D) The collinearity of DRM2 genes in different species. (E) The collinearity of UBP26 genes in different species. (F) The collinearity of NRPD1 genes in different species. (G) The collinearity of NRPE5 genes in different species. $(H)$ The collinearity of MET1 genes in different species. The species name are Chlamydomonas reinhardtii (Cr), Physcomitrella patens (Pp), Selaginella moellendorffii (Sm), Amborella trichopoda (Atr), Zea mays (Zm), Sorghum bicolor (Sb), Oryza sativa (Os), Hordeum vulgare (Hv), Solanum lycopersicum (SI), Vitis vinifera $(\mathrm{Vv})$, Citrullus lanatus (Cl), Phaseolus vulgaris (Pv), Populus trichocarpa (Pt), Gossypium raimondii (Gr), Theobroma cacao (Tc), Carica papaya (Cp), Arabidopsis thaliana (At). (PDF 584 kb)

Additional file 2: Figure S2. Exon-intron structures and domain structures. (A-DD) Exon-intron structures and domain structures of RDR2, CLSY1, DCL3, HEN1, AGO4, RDM1, DRD1, MORC6, SUVH2, SUVH9, KTF1, DRM1, DRM2, JMJ14, UBP26, HDA6, SUVH4, IDN2, IDP1, NRPD1, NRPE1, NRPD/E2, NRPD/E4, NRPE5, CMT2, CMT3, DDM, DME, ROS1 and DML3. a: A summary of gene length, exon number, exon length and intron length of gene. The green and yellow in the table represent genes with extreme values. b: The gene structure was shown by the online tool GSDS. c: The domain structures were shown by the online tool SMART. The species name are Chlamydomonas reinhardtii ( $\mathrm{Cr}$ ), Physcomitrella patens (Pp), Selaginella moellendorffii (Sm), Amborella trichopoda (Atr), Zea mays (Zm), Sorghum bicolor (Sb), Oryza sativa (Os), Hordeum vulgare (Hv), Solanum lycopersicum (SI), Vitis vinifera (VV), Citrullus lanatus (Cl), Phaseolus vulgaris (Pv), Populus trichocarpa (Pt), Gossypium raimondii (Gr), Theobroma cacao (Tc), Carica papaya (Cp), Arabidopsis thaliana (At). (PDF 4727 kb)

Additional file 3: Figure S3. The phylogenetic tree of methylationrelated genes constructed by a neighbor-joining method in 18 species. (A-S) The evolutionary tree of RDR2, DCL3, CLSY1, HEN1, RDM1, DRD1, 
DMS3, SUVH2/9, KTF1, JMJ14, UBP26, HDA6, SUVH4, IDN2, IDP1, NRPD1_NRPE1, NRPD/E2, NRPD/E4, NRPE5, and DME_ROS1_DML3 genes. (PDF $824 \mathrm{~kb}$ )

Additional file 4: Table S1. The gene ID of methylation-related genes in 77 species. (XLSX $13399 \mathrm{~kb}$ )

Additional file 5: Table S2. In 77 species, the number of methylationrelated genes, the number of single-copy genes, the number of multicopy genes, the number of missing genes, and the chromosome ploidy of the sequenced species. (XLSX $58 \mathrm{~kb}$ )

Additional file 6: Table S3. In six polyploid plants, the normalized number of methylation-related genes, the normalized number of singlecopy genes, the normalized number of multi-copy genes, the normalized number of missing genes, and the chromosome ploidy of the sequenced species. (XLSX $15 \mathrm{~kb}$ )

Additional file 7: Table S4. A summary of the duplication models of some methylation-related genes in five species. (XLSX $44 \mathrm{~kb}$ )

Additional file 8: Table S5. The gene length, exon number, exon length, intron length of methylation-related genes in 18 species. A: Gene length of methylation-related genes in 18 species. B: The number of genes with extreme values in gene length. C: Exon number of methylation-related genes in 18 species. D: The number of genes with extreme values in exon number. E: Gene length of methylation-related genes in 18 species. F: The number of genes with extreme values in exon length. G: Intron length of methylation-related genes in 18 species. $\mathrm{H}$ : The number of genes with extreme values in intron length. (XLSX $70 \mathrm{~kb}$ )

Additional file 9: Table S6. Summary of 77 sequenced genomes used for identification of methylation-related genes. (XLSX $15 \mathrm{~kb}$ )

Additional file 10: Table S7. Gene names used in gene structure analysis and phylogenetic analysis. (XLSX $72 \mathrm{~kb}$ )

Additional file 11: Table S8. The SRA accession number of RNA-Seq data used in this study. (XLSX $31 \mathrm{~kb})$

\section{Abbreviations}

CDS: Coding sequences; GFF3: General Feature Format 3;

Ka: nonsynonymous substitutions per nonsynonymous site; Ks: synonymous substitutions per synonymous site; RdDM: RNA-directed DNA methylation; siRNAs: small interfering RNAs; WGD: Whole genome duplication

\section{Acknowledgements}

Not applicable

\section{Authors' contributions}

MW and XZ conceived the experiment. LP, LZ, JL, CS and PQ performed bioinformatics analysis and analyzed the results. LP wrote the manuscript draft, MW and LT revised it. All authors read and approved the final manuscript.

\section{Funding}

This project was financially supported by the National Natural Science Foundation of China (31801405) and Young Elite Scientists Sponsorship Program by China Association for Science and Technology to MW. The funding bodies had no role in the design of the study, collection, analysis, and interpretation of data, and in writing the manuscript.

\section{Availability of data and materials}

All data analysed during this study are included in this published article and its supplementary information files.

\section{Ethics approval and consent to participate}

Not applicable.

\section{Consent for publication \\ Not applicable.}

\section{Competing interests}

The authors declare that they have no competing interests.
Received: 20 March 2019 Accepted: 3 July 2019

Published online: 12 July 2019

\section{References}

1. Bender J. DNA methylation and epigenetics Annu Rev Plant Biol.200455:41-68.

2. Goll MG, Bestor TH. Eukaryotic cytosine methyltransferases. Annu Rev Plant Biol. 2005:74:481-514.

3. Feng S, Cokus SJ, Zhang X, Chen PY, Bostick M, Goll MG, Hetzel J, Jain J, Strauss $\mathrm{SH}$, Halpern ME, et al. Conservation and divergence of methylation patterning in plants and animals. Proc Natl Acad Sci U S A. 2010;107:8689-94.

4. Zemach A, McDaniel IE, Silva P, Zilberman D. Genome-wide evolutionary analysis of eukaryotic DNA methylation. Science. 2010;328:916-9.

5. Matzke MA, Mosher RA. RNA-directed DNA methylation: an epigenetic pathway of increasing complexity. Nat Rev Genet. 2014;15:394-408.

6. Jullien $\mathrm{PE}$, Berger F. DNA methylation reprogramming during plant sexual reproduction? Trends Genet. 2010;26:394-9.

7. Bauer MJ, Fischer RL. Genome demethylation and imprinting in the endosperm. Curr Opin Plant Biol. 2011;14:162-7.

8. Cedar H, Bergman Y. Programming of DNA methylation patterns. Annu Rev Biochem. 2012:81:97-117.

9. Diez CM, Roessler K, Gaut BS. Epigenetics and plant genome evolution. Curr Opin Plant Biol. 2014:18:1-8.

10. Heard E, Martienssen RA. Transgenerational epigenetic inheritance: myths and mechanisms. Cell. 2014:157:95-109.

11. Zhong X. Comparative epigenomics: a powerful tool to understand the evolution of DNA methylation. New Phytol. 2016:210:76-80.

12. Cao X, Aufsatz W, Zilberman D, Mette MF, Huang MS, Matzke M, Jacobsen SE. Role of the DRM and CMT3 methyltransferases in RNA-directed DNA methylation. Curr Biol. 2003;13:2212-7.

13. Henderson IR, Deleris A, Wong W, Zhong X, Chin HG, Horwitz GA, Kelly KA, Pradhan S, Jacobsen SE. The De novo cytosine methyltransferase DRM2 requires intact UBA domains and a catalytically mutated paralog DRM3 during RNAdirected DNA methylation in Arabidopsis thaliana. PLoS Genet. 2010;6:e1001182.

14. Stroud H, Greenberg MV, Feng S, Bernatavichute YV, Jacobsen SE. Comprehensive analysis of silencing mutants reveals complex regulation of the Arabidopsis methylome. Cell. 2013;152:352-64.

15. Law JA, Jacobsen SE. Establishing, maintaining and modifying DNA methylation patterns in plants and animals. Nat Rev Genet. 2010;11:204-20.

16. Ma L, Hatlen A, Kelly LJ, Becher H, Wang W, Kovarik A, Leitch IJ, Leitch AR. Angiosperms are unique among land plant lineages in the occurrence of key genes in the RNA-directed DNA methylation (RdDM) pathway. Genome Biol Evol. 2015:7:2648-62.

17. Cao X, Jacobsen SE. Locus-specific control of asymmetric and CpNpG methylation by the DRM and CMT3 methyltransferase genes. Proc Natl Acad Sci U S A. 2002;99(Suppl 4):16491-8

18. Zemach A, Kim MY, Hsieh PH, Coleman-Derr D, Eshed-Williams L, Thao K Harmer SL, Zilberman D. The Arabidopsis nucleosome remodeler DDM1 allows DNA methyltransferases to access $\mathrm{H} 1$-containing heterochromatin. Cell. 2013;153:193-205.

19. Ryan DP, Owen-Hughes T. Snf2-family proteins: chromatin remodellers for any occasion. Curr Opin Chem Biol. 2011;15:649-56.

20. Le Masson I, Jauvion V, Bouteiller N, Rivard M, Elmayan T, Vaucheret H. Mutations in the Arabidopsis H3K4me2/3 demethylase JMJ14 suppress posttranscriptional gene silencing by decreasing transgene transcription. Plant Cell. 2012:24:3603-12

21. Liu X, Luo M, Wu K. Epigenetic interplay of histone modifications and DNA methylation mediated by HDA6. Plant Signal Behav. 2012;7:633-5.

22. Sridhar W, Kapoor A, Zhang K, Zhu J, Zhou T, Hasegawa PM, Bressan RA, Zhu JK. Control of DNA methylation and heterochromatic silencing by histone H2B deubiquitination. Nature. 2007;447:735-8.

23. Johnson LM, Bostick M, Zhang X, Kraft E, Henderson I, Callis J, Jacobsen SE. The SRA methyl-cytosine-binding domain links DNA and histone methylation. Curr Biol. 2007;17:379-84.

24. Herr AJ, Jensen M, Dalmay T, Baulcombe DC. RNA polymerase IV directs silencing of endogenous DNA. Science. 2005;308:118-20.

25. Onodera Y, Haag JR, Ream T, Costa Nunes P, Pontes O, Pikaard CS. Plant nuclear RNA polymerase IV mediates siRNA and DNA methylationdependent heterochromatin formation. Cell. 2005;120:613-22.

26. Haag JR, Pikaard CS. Multisubunit RNA polymerases IV and V: purveyors of non-coding RNA for plant gene silencing. Nat Rev Mol Cell Biol. 2011:12: 483-92. 
27. Tucker SL, Reece J, Ream TS, Pikaard CS. Evolutionary history of plant multisubunit RNA polymerases IV and V: subunit origins via genome-wide and segmental gene duplications, retrotransposition, and lineage-specific subfunctionalization. Cold Spring Harb Symp Quant Biol. 2010;75:285-97.

28. Haag JR, Ream TS, Marasco M, Nicora CD, Norbeck AD, Pasa-Tolic L, Pikaard CS. In vitro transcription activities of pol IV, pol V, and RDR2 reveal coupling of pol IV and RDR2 for dsRNA synthesis in plant RNA silencing. Mol Cell. 2012:48:811-8

29. Smith LM, Pontes O, Searle I, Yelina N, Yousafzai FK, Herr AJ, Pikaard CS, Baulcombe DC. An SNF2 protein associated with nuclear RNA silencing and the spread of a silencing signal between cells in Arabidopsis. Plant Cell. 2007;19:1507-21.

30. Xie Z, Johansen LK, Gustafson AM, Kasschau KD, Lellis AD, Zilberman D, Jacobsen SE, Carrington JC. Genetic and functional diversification of small RNA pathways in plants. PLoS Biol. 2004;2:e104.

31. Ji L, Chen X. Regulation of small RNA stability: methylation and beyond. Cell Res. 2012;22:624-36.

32. Havecker ER, Wallbridge LM, Hardcastle TJ, Bush MS, Kelly KA, Dunn RM, Schwach F, Doonan JH, Baulcombe DC. The Arabidopsis RNA-directed DNA methylation argonautes functionally diverge based on their expression and interaction with target loci. Plant Cell. 2010;22:321-34.

33. Dong L, Liu M, Fang YY, Zhao JH, He XF, Ying XB, Zhang YY, Xie Q, Chua $\mathrm{NH}$, Guo HS. DRD1-pol V-dependent self-silencing of an exogenous silencer restricts the non-cell autonomous silencing of an endogenous target gene. Plant J. 2011;68:633-45.

34. Zhong X, Hale CJ, Law JA, Johnson LM, Feng S, Tu A, Jacobsen SE. DDR complex facilitates global association of RNA polymerase $V$ to promoters and evolutionarily young transposons. Nat Struct Mol Biol. 2012;19:870-5.

35. Moissiard G, Cokus SJ, Cary J, Feng S, Billi AC, Stroud H, Husmann D, Zhan Y, Lajoie BR, McCord RP, et al. MORC family ATPases required for heterochromatin condensation and gene silencing. Science. 2012;336:1448-51.

36. Iyer LM, Anantharaman V, Wolf MY, Aravind L. Comparative genomics of transcription factors and chromatin proteins in parasitic protists and other eukaryotes. Int J Parasitol. 2008;38:1-31.

37. Liu ZW, Shao CR, Zhang CJ, Zhou JX, Zhang SW, Li L, Chen S, Huang HW, Cai T, He XJ. The SET domain proteins SUVH2 and SUVH9 are required for pol V occupancy at RNA-directed DNA methylation loci. PLoS Genet. 2014; 10:e1003948.

38. Bies-Etheve N, Pontier D, Lahmy S, Picart C, Vega D, Cooke R, Lagrange T. RNA-directed DNA methylation requires an AGO4-interacting member of the SPT5 elongation factor family. EMBO Rep. 2009;10:649-54.

39. Zhang CJ, Ning YQ, Zhang SW, Chen Q, Shao CR, Guo YW, Zhou JX, Li L, Chen S, He XJ. IDN2 and its paralogs form a complex required for RNAdirected DNA methylation. PLoS Genet. 2012:8:e1002693.

40. Zhu JK. Active DNA demethylation mediated by DNA glycosylases. Annu Rev Genet. 2009;43:143-66

41. Gong ZZ, Morales-Ruiz T, Ariza RR, Roldan-Arjona T, David L, Zhu JK. ROS1, a repressor of transcriptional gene silencing in Arabidopsis, encodes a DNA glycosylase/lyase. Cell. 2002;111:803-14

42. Choi Y, Gehring M, Johnson L, Hannon M, Harada JJ, Goldberg RB, Jacobsen SE, Fischer RL. DEMETER, a DNA glycosylase domain protein, is required for endosperm gene imprinting and seed viability in Arabidopsis. Cell. 2002;110:33-42.

43. Tedeschi F, Rizzo P, Huong BTM, Czihal A, Rutten T, Altschmied L, Scharfenberg S, Grosse I, Becker C, Weigel D, et al. EFFECTOR OF TRANSCRIPTION factors are novel plant-specific regulators associated with genomic DNA methylation in Arabidopsis. New Phytol. 2019;221:261-78.

44. Thomas M, Pingault L, Poulet A. Evolutionary history of methyltransferase 1 genes in hexaploid wheat. BMC Genomics. 2014;15:922.

45. Huang Y, Kendall T, Forsythe ES, Dorantes-Acosta A, Li S, Caballero-Perez J, Chen X, Arteaga-Vazquez M, Beilstein MA, Mosher RA. Ancient origin and recent innovations of RNA polymerase IV and V. Mol Biol Evol. 2015;32: 1788-99.

46. Bewick AJ, Niederhuth CE, Ji L, Rohr NA, Griffin PT, Leebens-Mack J, Schmitz RJ. The evolution of CHROMOMETHYLASES and gene body DNA methylation in plants. Genome Biol. 2017;18:65.

47. Lu F, Cui X, Zhang S, Liu C, Cao X. JMJ14 is an H3K4 demethylase regulating flowering time in Arabidopsis. Cell Res. 2010;20:387-90.

48. Schmitz RJ, Tamada Y, Doyle MR, Zhang X, Amasino RM. Histone H2B deubiquitination is required for transcriptional activation of FLOWERING LOCUS C and for proper control of flowering in Arabidopsis. Plant Physiol. 2009;149:1196-204.
49. Tanaka M, Kikuchi A, Kamada H. The Arabidopsis histone deacetylases HDA6 and HDA19 contribute to the repression of embryonic properties after germination. Plant Physiol. 2008;146:149-61.

50. Grafi G, Ben-Meir H, Avivi Y, Moshe M, Dahan Y, Zemach A. Histone methylation controls telomerase-independent telomere lengthening in cells undergoing dedifferentiation. Dev Biol. 2007;306:838-46.

51. Schmutz J, Cannon SB, Schlueter J, Ma J, Mitros T, Nelson W, Hyten DL, Song Q, Thelen JJ, Cheng J, et al. Genome sequence of the palaeopolyploid soybean. Nature. 2010;463:178-83.

52. Velasco R, Zharkikh A, Affourtit J, Dhingra A, Cestaro A, Kalyanaraman A, Fontana P, Bhatnagar SK, Troggio M, Pruss D, et al. The genome of the domesticated apple (Malus x domestica Borkh). Nat Genet. 2010;42:833-9.

53. Tang $H$, Bowers JE, Wang $X$, Paterson AH. Angiosperm genome comparisons reveal early polyploidy in the monocot lineage. Proc Natl Acad Sci U S A. 2010;107:472-7.

54. Bowers JE, Chapman BA, Rong J, Paterson AH. Unravelling angiosperm genome evolution by phylogenetic analysis of chromosomal duplication events. Nature. 2003;422:433-8.

55. Bernatavichute $\mathrm{Y}$, Zhang X, Cokus S, Pellegrini M, Jacobsen SE. Genomewide association of histone $\mathrm{H} 3$ lysine nine methylation with CHG DNA methylation in Arabidopsis thaliana. PLoS One. 2008;3:e3156.

56. Bewick AJ, Ji L, Niederhuth CE, Willing EM, Hofmeister BT, Shi X, Wang L, Lu $Z$, Rohr NA, Hartwig B, et al. On the origin and evolutionary consequences of gene body DNA methylation. Proc Natl Acad Sci U S A. 2016;113:9111-6.

57. Niederhuth CE, Bewick AJ, Ji L, Alabady MS, Kim KD, Li Q, Rohr NA, Rambani A, Burke JM, Udall JA, et al. Widespread natural variation of DNA methylation within angiosperms. Genome Biol. 2016;17:194.

58. Maher C, Stein L, Ware D. Evolution of Arabidopsis microRNA families through duplication events. Genome Res. 2006;16:510-9.

59. Wang $Y$, Tang H, Debarry JD, Tan X, Li J, Wang X, Lee TH, Jin H, Marler B, Guo $H$, et al. MCScanX: a toolkit for detection and evolutionary analysis of gene synteny and collinearity. Nucleic Acids Res. 2012;40:e49.

60. Larkin MA, Blackshields G, Brown NP, Chenna R, McGettigan PA, McWilliam H, Valentin F, Wallace IM, Wilm A, Lopez R, et al. Clustal W and Clustal X version 2.0. Bioinformatics. 2007;23:2947-8.

61. Felsenstein J. PHYLIP-phylogeny inference package (version 3.2). Cladistics. 1989:5:164-6.

62. Suyama M, Torrents D, Bork P. PAL2NAL: robust conversion of protein sequence alignments into the corresponding codon alignments. Nucleic Acids Res. 2006;34:W609-12.

63. Yang Z. PAML 4: phylogenetic analysis by maximum likelihood. Mol Biol Evol. 2007:24:1586-91.

64. Bolger AM, Lohse M, Usadel B. Trimmomatic: a flexible trimmer for illumina sequence data. Bioinformatics. 2014;30:2114-20.

65. Kim D, Langmead B, Salzberg SL. HISAT: a fast spliced aligner with low memory requirements. Nat Methods. 2015;12:357-60.

66. Pertea M, Pertea GM, Antonescu CM, Chang TC, Mendell JT, Salzberg SL. StringTie enables improved reconstruction of a transcriptome from RNA-seq reads. Nat Biotechnol. 2015;33:290-5.

\section{Publisher's Note}

Springer Nature remains neutral with regard to jurisdictional claims in published maps and institutional affiliations.

Ready to submit your research? Choose BMC and benefit from:

- fast, convenient online submission

- thorough peer review by experienced researchers in your field

- rapid publication on acceptance

- support for research data, including large and complex data types

- gold Open Access which fosters wider collaboration and increased citations

- maximum visibility for your research: over $100 \mathrm{M}$ website views per year

At $\mathrm{BMC}$, research is always in progress.

Learn more biomedcentral.com/submission 